\title{
原位裂解碳对氮化铝基微波衰减陶瓷性能的影响
}

\author{
何永钦 ${ }^{1,2}$, 李晓云 ${ }^{1}$, 张景贤 ${ }^{3}$, 李晓光 ${ }^{3}$
}

(1. 南京工业大学 材料科学与工程学院, 南京 210009; 2. 江苏先进无机功能复合材料协同创新中心, 南京 210009; 3. 中国科学院 上海硅酸盐研究所, 结构陶瓷工程研究中心, 上海 200050)

摘 要: 碳材料具有优异的吸波性能, 但是难以在陶瓷基体中均匀分散。本研究通过酚醛树脂裂解的方法在氮化铝 陶瓷基体中引入碳, 研究了酚醛树脂的添加量对氮化铝陶瓷烧结性能、微观形貌、导热性能和介电性能的影响。研 究发现, 酚醛裂解形成的碳能够有效促进氮化铝陶瓷的致密化进程, 降低烧结温度。当酚醛树脂含量为 $3 \mathrm{wt} \%, 1700^{\circ} \mathrm{C}$ 烧结后陶瓷的致密度达到 $99.26 \%$ 。此外, 裂解碳的引入能够显著提高材料的导热性能, 并在材料的气孔中和氮化 铝的晶界处形成碳膜, 从而显著提高材料的介电性能。当酚醛树脂含量为 $6 \mathrm{wt} \%$ 时, 材料热导率达 $135.1 \mathrm{~W} /(\mathrm{m} \cdot \mathrm{K})$, 在 $\mathrm{X}$ 波段的介电损耗为 0.3 , 表明材料具有良好的微波衰减能力, 有望应用于大功率的微波电真空器件中。

关 键 词: 酚醛树脂; 氮化铝; 热导率; 介电损耗

中图分类号: TQ174 文献标识码: A

\section{In situ Pyrolyzed Carbon on the Property of AlN-based Microwave Attenuation Ceramics}

\author{
HE Yong-Qin ${ }^{1,2}$, LI Xiao-Yun ${ }^{1}$, ZHANG Jing-Xian ${ }^{3}$, LI Xiao-Guang ${ }^{3}$
}

(1. School of Materials Science and Engineering, Nanjing Tech University, Nanjing 210009, China; 2. Jiangsu Collaborative Innovation Center for Advanced Inorganic Function Composites, Nanjing 210009, China; 3. Structural Ceramics Engineering Research Center, Shanghai Institute of Ceramics, Chinese Academy of Sciences, Shanghai 200050, China)

\begin{abstract}
Carbon materials exhibit excellent microwave absorbing properties. However, it is difficult to disperse carbon homogeneously in the ceramic substrate. In this study, carbon was introduced into AlN matrix by addition of phenolic resin followed by pyrolysis process. The effects of phenolic resin content on sintering behavior, microstructure, thermal conductivity and dielectric properties of AlN ceramics were investigated. It was found that phenolic resin derived carbon could effectively promote the densification of AlN ceramics and reduce the sintering temperature. Relative density of the AlN ceramics reached $99.26 \%$ after sintering at $1700^{\circ} \mathrm{C}$ with addition of $3 \mathrm{wt} \%$ phenolic resin. In addition, it was found that the presence of phenolic resin derived carbon can effectively improve the thermal conductivity of the ceramics. The dielectric properties were also improved due to the carbon films formed in the pores and at AIN grain boundaries. With addition of 6wt\% phenolic resin, AlN ceramics showed high thermal conductivity of $135.1 \mathrm{~W} /(\mathrm{m} \cdot \mathrm{K}$ ) and excellent microwave attenuation properties (the dielectric loss was 0.3 at X-band), which were perspective candidates for possible applications in high power microwave vacuum electron devices.
\end{abstract}

Key words: phenolic resin; AlN; thermal conductivity; dielectric loss

收稿日期：2017-04-28; 收到修改稿日期：2017-06-13

作者简介: 何永钦(1992-), 男, 硕士研究生. E-mail: 15261806381@163.com

通讯作者: 李晓云, 教授. E-mail: lixiaoyun@njtech.edu.cn 
微波衰减陶瓷被广泛应用于微波电真空器件中, 可以抑制自激振荡、消除有害的电磁模式和增强带 宽等, 确保电真空器件处于稳定的工作状态 ${ }^{[1]}$ 。传统 的微波衰减陶瓷大都采用氧化铝和氧化铍作为基体, 通过添加不同的衰减相制备而成, 随着大功率微波 电真空器件的发展以及人们对环境问题的关注, 这 类微波衰减陶瓷由于热导率低和有毒等不足而逐渐 被限制使用 ${ }^{[2]}$ 。

目前, 兼具高导热和良好衰减性能的微波衰减 陶瓷已经成为研究的焦点。氮化铝具有高热导率 ${ }^{[3]}$ 、 适中的介电系数、良好的化学和热稳定性以及很高 的电阻率, 适合作为微波衰减陶瓷的基体相。常见 的研究体系主要分为两类: 一类是 AlN-金属体系, 如 AlN-Mo ${ }^{[4]}$ 、AlN-W ${ }^{[5-6]}$ 复相陶瓷, 通过添加金属 颗粒作为衰减相来提高材料的微波衰减能力, 但是 由于金属和陶瓷密度相差大, 分散困难, 局部会出 现金属颗粒连通的情况，材料的绝缘性能难以控制; 另一类是 AlN-陶瓷体系, 常见的是 $\mathrm{AlN}-\mathrm{SiC}^{[7]}$ 陶瓷 体系。虽然该体系具有优异的微波衰减性能, 但是 由于固溶引起热导率下降, 使其在大功率器件中的 应用受到限制, 如 Serbenyuk 等 ${ }^{[8]}$ 制备的 AlN-SiC 陶 瓷, 热导率只有 $37 \sim 82 \mathrm{~W} /(\mathrm{m} \cdot \mathrm{K})$ 。研究发现, 碳材料 也是一种优良的微波衰减相, 一些学者通过添加石 墨或者炭黑研究了 AlN-C 复相陶瓷, 发现该体系既 具有良好的微波衰减性能 ${ }^{[9-10]}$, 又具有较高的热导 率, 有可能同时满足高热导率和高衰减性能的要 求。但是 C 在 AlN 基体中难以分散, 通常添加量较 高, 造成材料烧结困难, 性能离散性较大。为了提高 碳材料的分散均匀性, 同时为了降低碳颗粒的尺寸, 以最大限度地提高其微波衰减性能, 本工作采用添 加酚醛树脂作为碳源, 并综合研究了该方法所制备 的 AIN-C 复相陶瓷的烧结性能、微观结构、热学性 能和介电性能与酚醛树脂含量的关系。

\section{1 实验方法}

以 $\operatorname{AlN}\left(\mathrm{F}\right.$ 级, 德山公司, 日本)为陶瓷粉体, $\mathrm{Y}_{2} \mathrm{O}_{3}$ (上海跃凯金属材料有限公司, 上海)为烧结助剂, 含量固定为 $3 \mathrm{wt} \%$, 酚醛树脂 (上海祁南胶粘材料厂, 上海)为碳源，含量分别为 $0.5 \mathrm{wt} \% 、 1.5 \mathrm{wt} \% 、 3 \mathrm{wt} \%$ 、 $5 \mathrm{wt} \%$ 和 $6 \mathrm{wt} \%$ 。使用乙醇 $($ 上海凌峰化学试剂有限公 司, 上海)作为研磨介质, 球磨转速为 $380 \mathrm{r} / \mathrm{min}$, 球 磨 $4 \mathrm{~h}$ 。将球磨后的浆料在 $60^{\circ} \mathrm{C}$ 干燥 $24 \mathrm{~h}$, 然后研 磨、过 $74 \mu \mathrm{m}$ 篮。将所得粉体先在 $20 \mathrm{MPa}$ 预压成 型, 再在 $200 \mathrm{MPa}$ 冷等静压获得陶瓷素坏, 然后将 素坏放在涂有 $\mathrm{BN}$ 的石墨坩埚中, 氮气气氛下, 分
别在 $1700^{\circ} \mathrm{C} 、 1750^{\circ} \mathrm{C}$ 和 $1800^{\circ} \mathrm{C}$ 保温 $2 \mathrm{~h}$, 常压烧结 制备得到复相陶瓷。

使用阿基米德排水法测试材料的相对密度。采 用 X 射线衍射仪(D8 Advance, Bruker, 德国)分析物 相组成, 扫描范围 $2 \theta=10^{\circ} \sim 80^{\circ}$, 扫描速率 $5^{\circ} / \mathrm{min}$ 。将 材料的断面蒸金后, 采用扫描电子显微镜(S-4800, Hitachi, 日本)观察其断面微观形貌, 并结合能谱分 析材料的元素组成。将材料加工成 $\phi 10 \mathrm{~mm} \times 2 \mathrm{~mm}$ 的圆片，采用激光热导仪 (LFA427 Nanoflash, Netzsch, 德国)测试材料室温下的热扩散系数。材料 的热导率 $\lambda$ 根据公式 $\lambda=\rho \times \alpha \times C_{\mathrm{p}}$ 计算, 其中 $\rho$ 为材料 的密度, $\alpha$ 为材料的热扩散系数, $C_{\mathrm{p}}$ 为材料的热容, 由于本实验材料中其他相的含量很少, 所以使用纯 AlN 的热容 $(0.75 \mathrm{~J} /(\mathrm{g} \cdot \mathrm{K}))^{[11]}$ 进行计算。将材料加工 成 $22.86 \mathrm{~mm} \times 10.16 \mathrm{~mm} \times 2 \mathrm{~mm}$ 的方片, 采用网络矢 量分析仪(VNA, N5225A, Agilent)测试材料在 X 波 段的介电常数, 材料的介电损耗 $\tan \delta$ 根据公式 $\tan \delta=\varepsilon^{\prime \prime} / \varepsilon^{\prime}$ 计算, 其中 $\varepsilon^{\prime \prime}$ 为材料的介电常数虚部, $\varepsilon^{\prime}$ 为 材料的介电常数实部。

\section{2 结果与讨论}

\section{1 烧结性能}

图 1 是酚醛树脂在高温下裂解的反应过程, 从 图中可以看出, 根据酚醛树脂碳碳键断开位置的不 同, 分别生成苯酚和甲酚, 然后亚甲基与羟基发生 反应导致碳氢交联，或者羟基官能团相互反应生成 醚键, 随着温度升高, 进一步发生脱氧脱氢反应, 逐渐转变为具有并苯结构的碳, 最终并苯结构的碳逐 渐脱氢形成碳材料。整个过程的气体产物以 $\mathrm{H}_{2} \mathrm{O}$ 和 $\mathrm{H}_{2}$ 为主, 其次是 $\mathrm{CH}_{4} 、 \mathrm{CO}$ 以及微量的 $\mathrm{CO}_{2}$ 和 $\mathrm{C}_{2} \mathrm{H}_{6}{ }^{[12]}$ 。

图 2 是不同温度烧结的复相陶瓷相对密度与酚

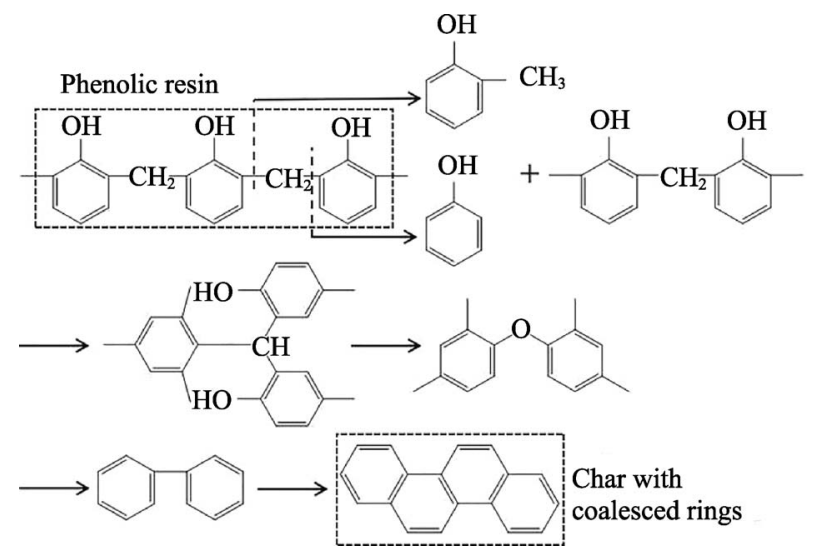

图 1 酚醛树脂的裂解反应过程

Fig. 1 Pyrolysis process of phenolic resin 


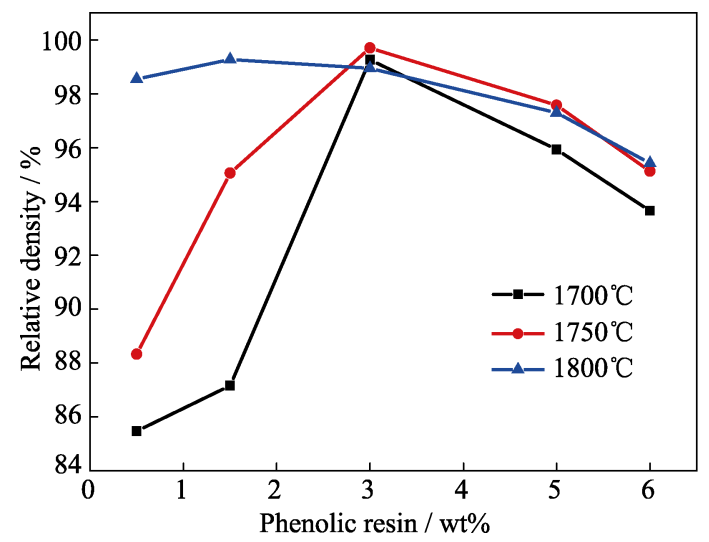

图 2 酚醛树脂含量对不同温度烧结的复相陶瓷相对密度的 影响

Fig. 2 Effects of phenolic resin content on the relative densities of composites sintered at different temperatures

醛树脂含量的关系图, 从图中可以看出, 当烧结温 度为 $1700^{\circ} \mathrm{C}$ 和 $1750^{\circ} \mathrm{C}$, 酚醛树脂含量为 $0.5 \mathrm{wt} \%$ 时, 材料的相对密度分别为 $85.47 \%$ 和 $88.33 \%$; 随着酚 醛树脂含量增加到 $3 \mathrm{wt} \%$ 时, 复相陶瓷的相对密度 逐渐提高到 99.26\%和 99.70\%，这表明添加酚醛树 脂可以有效地促进复相陶瓷的致密化进程，降低烧 结温度。这是因为酚醛树脂在高温下裂解形成的碳 会与 AlN 颗粒表面的氧化层发生碳热还原反应 ${ }^{[13]}$, 达到减少或消除氧化层的目的，从而有利于 AlN 的 传质, 促进复相陶瓷的烧结过程。当烧结温度为 $1800^{\circ} \mathrm{C}$ 时, 由于温度较高, 所以在酚酫树脂含量为 $0.5 \mathrm{wt} \%$ 时，材料就表现出较高的致密度，达到 $98.54 \%$ 。当酚醛树脂含量继续升高到 $5 \mathrm{wt} \%$ 及以上 时, 全部材料的致密度开始出现明显的下降, 这可 能是由于较多的残余碳阻碍了颗粒的重排, 从而阻 碍了复相陶瓷的致密化进程。

\section{2 物相与微观形貌}

图 3 为 $1800^{\circ} \mathrm{C}$ 烧结的复相陶瓷的 XRD 图谱, 从 图中可以看出, 材料中的主晶相为 AlN(65-0831)相, 次晶相的组成随着酚醛树脂含量发生变化。当酚醛 树脂含量为 $0.5 \mathrm{wt} \%$ 时, 次晶相为 $\mathrm{Y}_{3} \mathrm{Al}_{5} \mathrm{O}_{12}$ 相, 此时 AlN 颗粒表面的氧化物含量较高, 将与烧结助剂 $\mathrm{Y}_{2} \mathrm{O}_{3}$ 发生以下反应:

$$
5 \mathrm{Al}_{2} \mathrm{O}_{3}+3 \mathrm{Y}_{2} \mathrm{O}_{3}=2 \mathrm{Y}_{3} \mathrm{Al}_{5} \mathrm{O}_{12}
$$

随着酚醛树脂含量的增加, 残余碳通过碳热还 原反应逐渐消耗 $\mathrm{AlN}$ 颗粒表面的 $\mathrm{Al}_{2} \mathrm{O}_{3}$, 使得液相 产物逐渐转变为 $\mathrm{AlYO}_{3}$ 和 $\mathrm{Al}_{2} \mathrm{Y}_{4} \mathrm{O}_{9}$, 具体反应如下:

$$
\begin{aligned}
\mathrm{Al}_{2} \mathrm{O}_{3}+\mathrm{Y}_{2} \mathrm{O}_{3} & =2 \mathrm{AlYO}_{3} \\
\mathrm{Al}_{2} \mathrm{O}_{3}+2 \mathrm{Y}_{2} \mathrm{O}_{3} & =\mathrm{Al}_{2} \mathrm{Y}_{4} \mathrm{O}_{9}
\end{aligned}
$$

当酚醛树脂含量增加到 $5 \mathrm{wt} \%$ 时，此时材料中 的次晶相只有 $\mathrm{Y}_{2} \mathrm{O}_{3}$ 相, 一方面残余碳进一步消耗 氧化层, 使得烧结助剂 $\mathrm{Y}_{2} \mathrm{O}_{3}$ 过量; 另一方面, 残余

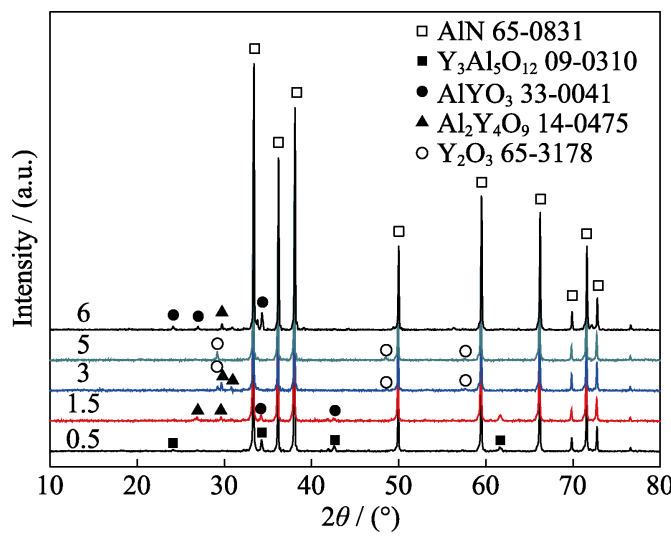

图 $31800^{\circ} \mathrm{C}$ 烧结的不同酚醛树脂含量复相陶瓷的 XRD 图谱

Fig. 3 XRD patterns of composites with different phenolic resin contents sintered at $1800^{\circ} \mathrm{C}$

碳将与 $\mathrm{Al}_{2} \mathrm{Y}_{4} \mathrm{O}_{9}$ 相发生反应, 使其转化为 $\mathrm{AlN}$ 和 $\mathrm{Y}_{2} \mathrm{O}_{3}$, 具体反应如下 ${ }^{[13]}$ :

$\mathrm{Al}_{2} \mathrm{Y}_{4} \mathrm{O}_{9}+\mathrm{N}_{2}+3 \mathrm{C}=2 \mathrm{AlN}+2 \mathrm{Y}_{2} \mathrm{O}_{3}+3 \mathrm{CO}$

当酚醛树脂含量进一步增加到 $6 \mathrm{wt} \%$ 时, 次晶 相中出现微量 $\mathrm{AlYO}_{3}$ 和 $\mathrm{Al}_{2} \mathrm{Y}_{4} \mathrm{O}_{9}$ 相, 可能由于残余 碳与表面氧化层的反应为固相反应，因残余碳在烧 结过程中会移动到三岔晶界位置, 无法与 AlN 颗粒 表面充分接触，从而使得碳对氧化层的消除作用 变弱。

图 4 为 $1800^{\circ} \mathrm{C}$ 烧结的复相陶瓷的 SEM 照片, 图 中深色部分为 $\mathrm{AlN}$ 晶粒, 呈现出典型的等轴晶形貌, 浅色部分为第二相, 主要分布在 AlN 晶粒的晶界和 三岔晶界处。从图 4 可以看出, AlN 晶粒尺寸较小, 并且分布比较均匀。在 AlN 陶瓷的液相烧结过程中, 晶界会逐渐向晶粒长大的方向移动，此时处于晶界 和三岔晶界上的第二相会阻碍晶界的移动, 晶粒进 一步长大则必须克服第二相形成的势垒 ${ }^{[14]}$, 从而消 耗更多的能量。因此处于晶界和三岔晶界处的第二 相会成为晶粒长大的障碍, 抑制晶粒的快速生长, 从而获得更加细小均匀的微结构。当酚醛树脂含量 较低时(0.5wt\%和 $1.5 \mathrm{wt} \%)$ 时，材料中几乎观察不到 明显的气孔，表现出很高的致密度。当酚醛树脂含 量提高到 $3 \mathrm{wt} \%$ 及以上时, 复相陶瓷中开始出现少 量的气孔, 并且在酚醛树脂含量为 $6 \mathrm{wt} \%$ 时, 材料中 能够明显观察到薄膜状的物质存在于气孔中、AlN 晶粒之间以及三岔晶界处。

图 5 为酚酫树脂含量为 $6 \mathrm{wt} \%$ 时复相陶瓷的 SEM 及元素分析, 从图 5(a)可以看出, 白色箭头所 指即为薄膜物质, 主要分布在三岔晶界处, 并将晶 粒相互连接起来, 同时伴随有明显的气孔。图 5(b) 为点 1 处的元素分析, 可以看出此处主要含有 $\mathrm{Al}$ 和 

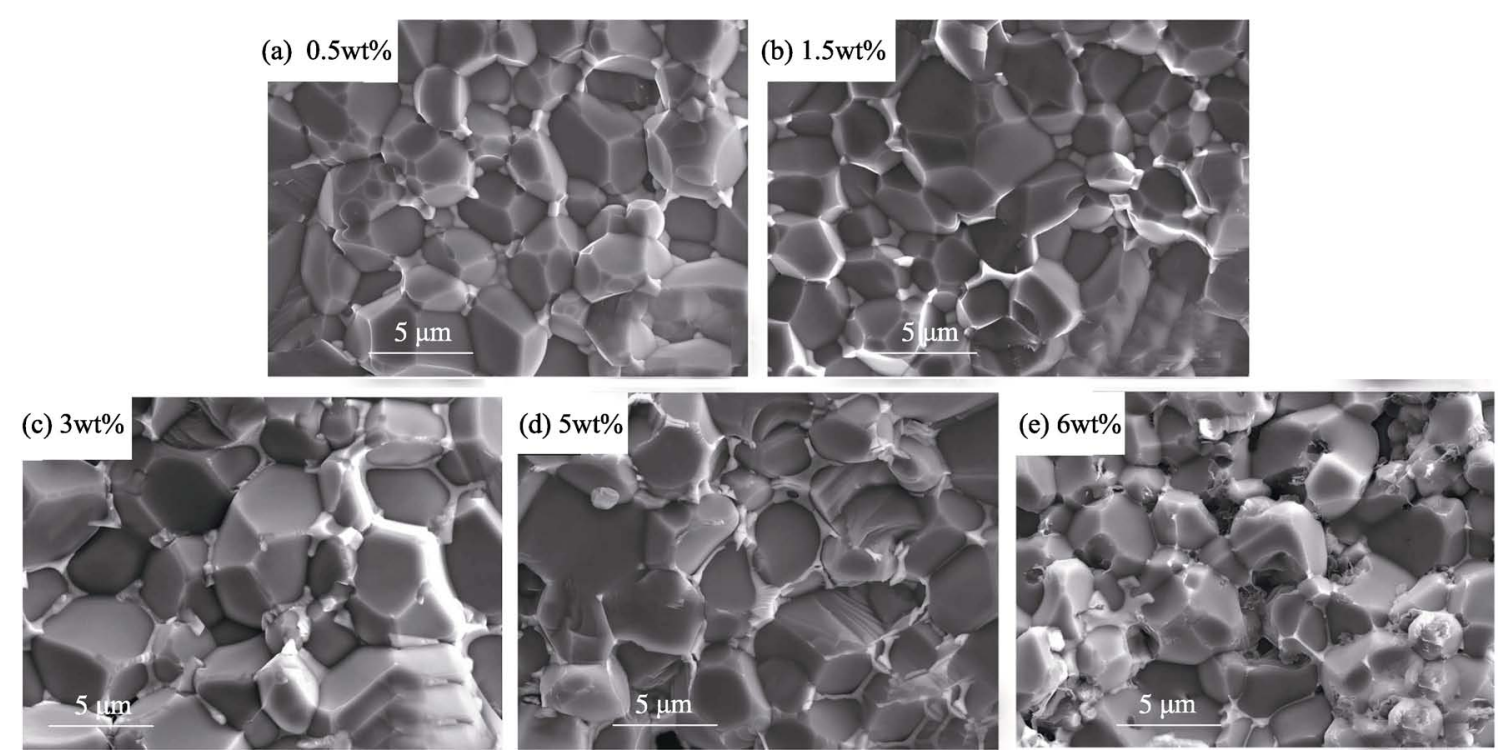

图 4 不同酚醛树脂含量复相陶瓷 $1800^{\circ} \mathrm{C}$ 烧结后的断面 SEM 照片及相应的 EDS 分析

Fig. 4 SEM images and EDS analysis of composites with different phenolic resin content sintered at $1800^{\circ} \mathrm{C}$
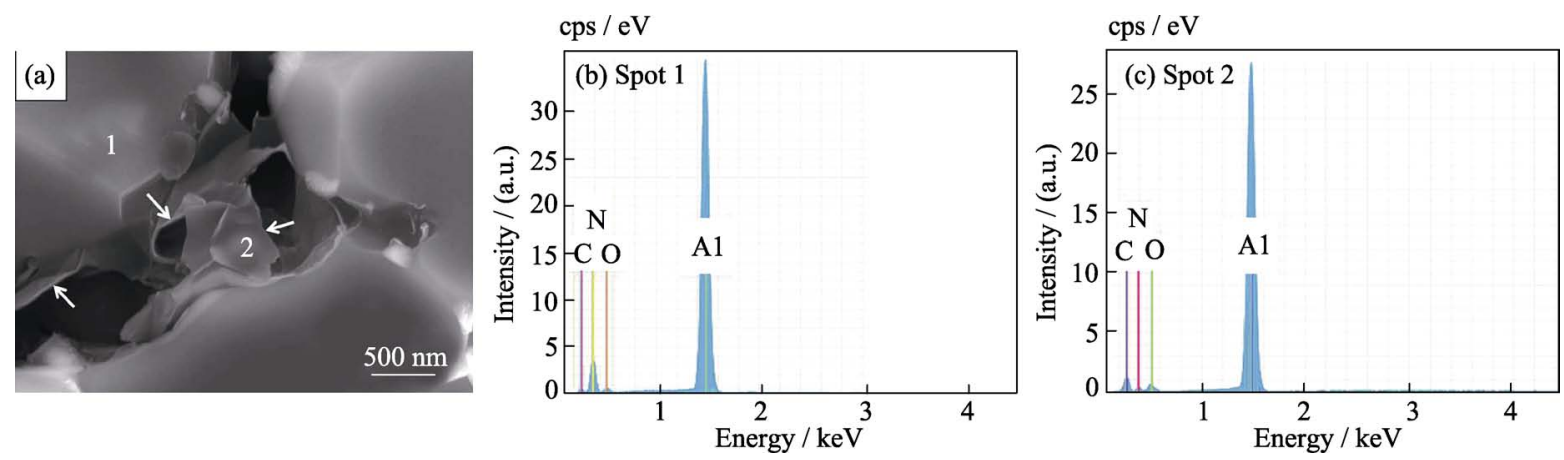

图 5 酚醛树脂含量为 $6 \mathrm{wt} \%$ 的复相陶瓷的 SEM 照片(a)及元素分析(b, c)结果

Fig. 5 SEM image (a) and EDS analysis (b, c) of the composite with $6 \mathrm{wt} \%$ phenolic resin

$\mathrm{N}$ 元素，表明该晶粒为氮化铝晶粒。图 5(c)为点 2 处的元素分析, 可以看出该处 C 元素的含量大幅度 升高, 可以推测该薄膜物质是酚醛树脂裂解后留下 的残余碳膜。这表明当酚醛树脂含量提高到 $6 \mathrm{wt} \%$ 时, 残余碳过剩, 过量的残余碳将会以碳膜的形式 存在于氮化铝晶粒之间和三岔晶界处, 在烧结过程 中晶界处的碳膜会阻碍氮化铝晶粒的生长, 并且形 成难以排出的气孔, 从而引起材料致密度逐渐下降。

\section{3 酚醛树脂含量对复相陶瓷热导率的影响}

由于微波衰减陶瓷在使用过程中会将电磁能逐 渐转化为热能, 材料具备优良的导热性能对于大功 率微波电真空器件的正常运转具有重要意义, 所以 有必要对复相陶瓷的热导率进行研究。图 6 是酚醛 树脂含量对 $1800^{\circ} \mathrm{C}$ 烧结的复相陶瓷热扩散系数和 热导率的影响, 从图中可以看出, 当酚醛树脂含量 从 $0.5 \mathrm{wt} \%$ 提高到 $3 \mathrm{wt} \%$ 时, 材料的热扩散系数和热 导率分别从 $58.03 \mathrm{~mm} / \mathrm{s}$ 和 $141.60 \mathrm{~W} /(\mathrm{m} \cdot \mathrm{K})$ 提高到 $68.81 \mathrm{~mm}^{2} / \mathrm{s}$ 和 $165.80 \mathrm{~W} /(\mathrm{m} \cdot \mathrm{K})$, 表明酚醛树脂对于
材料的导热性能具有显著的提升效果。影响 AlN 陶 瓷导热性能最重要的因素之一就是材料中的氧杂质, 氧杂质主要以两种形态存在于 AlN 粉体原料中, 第 一种是在 AlN 粉体表面形成很薄的非晶氧化层, 氧 化层的存在将会影响声子的传播速率, 对材料的导 热性能提高不利; 第二种是部分氧杂质会固溶入 AlN 的晶格中, 形成晶格氧缺陷, 具体反应如下 ${ }^{[15]}$ :

$$
\mathrm{Al}_{2} \mathrm{O}_{3} \rightarrow 2 \mathrm{Al}_{\mathrm{Al}}+3 \mathrm{O}_{\mathrm{N}}+\mathrm{V}_{\mathrm{Al}}
$$

晶格氧缺陷的形成将会引起 AlN 晶格中铝空位 含量的增加, 而铝空位具有很大的散射截面, 从而 造成声子传播过程中强烈的散射现象, 严重降低材 料的导热性能。在稀土烧结助剂存在的情况下, 添 加酚醛树脂后, 残余碳将会通过碳热还原反应消耗 掉 AIN 颗粒表面的氧化层, 使得参与液相反应的氧 杂质浓度逐渐降低, 从而推动反应(5)向逆反应方向 进行, 使得 AIN 晶格中的氧杂质含量降低, 所以材 料的热导率逐渐提高。当酚醛树脂含量继续提高到 $5 \mathrm{wt} \%$ 时, 材料的热扩散系数和热导率开始出现明 显的下降, 主要是由于材料的致密度开始降低, 存 


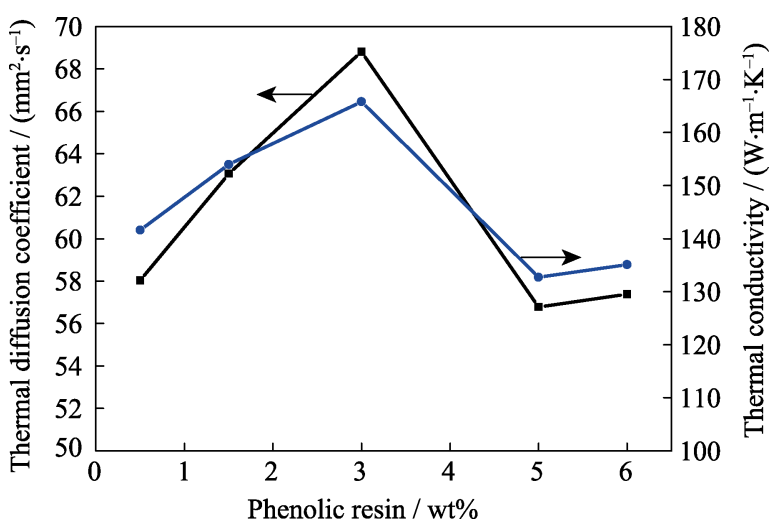

图 $61800^{\circ} \mathrm{C}$ 烧结的不同酚醛树脂含量复相陶瓷的导热性能 Fig. 6 Thermal properties of composites with different phenolic resin contents sintered at $1800^{\circ} \mathrm{C}$

在的气孔严重影响声子的传播。当酚醛树脂含量进 一步提高到 $6 \mathrm{wt} \%$ 时, 材料的致密度更低, 但是材料 的热导率并没有下降, 反而从 $132.7 \mathrm{~W} /(\mathrm{m} \cdot \mathrm{K})$ 略微 提高到 $135.1 \mathrm{~W} /(\mathrm{m} \cdot \mathrm{K})$ 。这可能是由于材料中的碳薄 膜填充在陶瓷的气孔之中, 将原本被气孔隔绝的氮 化铝晶粒连接起来。而碳材料的热导率较高, 当声 子传播遇到气孔时，能够起到桥梁作用，大大降低 气孔对声子传播的影响，因而材料的热导率没有出 现明显的恶化现象, 反而略有提高。较高的热导率 不仅有利于热能的散出, 维持器件正常的运转, 而 且对改善材料的抗热震性能有利。从图 4 和图 5 可 以看出, 碳膜主要分布在氮化铝晶粒之间和三岔晶 界处，而碳和氮化铝热膨胀系数不同容易引入局部 热应力, 对材料的宏观力学性能和抗热震性能存在 影响。但碳和氮化铝晶粒界面结合较弱, 对应力有 一定的消除作用。碳的含量对于材料的力学和抗热 震性能的影响规律正在进一步研究中。

\section{4 酚醛树脂含量对复相陶瓷介电性能的 影响}

复介电常数 $\left(\varepsilon=\varepsilon^{\prime}-j \varepsilon^{\prime \prime}\right)$ 通常用来预测材料微波衰 减能力的大小, 是设计微波衰减陶瓷的重要参数, 其中介电常数实部 $\varepsilon^{\prime}$ 代表材料弛豫极化的能力, 介 电常数虚部 $\varepsilon^{\prime \prime}$ 代表材料的介电损耗能力 ${ }^{[16]}$ 。复相陶 瓷在 X波段的介电性能与酚醛树脂含量的关系如图 7 所示，当酚醛树脂的含量在 3wt\%以下时，材料的 介电常数实部和虚部变化很小, 介电常数实部在 8.0 8.4 之间, 介电常数虚部在 $0.06 \sim 0.25$ 之间, 介电损 耗最高为 0.028 , 表现出较差的微波衰减能力。当 酚醛树脂含量提高到 $5 \mathrm{wt} \%$ 和 $6 \mathrm{wt} \%$ 时，材料的介 电常数实部和虚部显著提高, 在 $\mathrm{X}$ 波段的介电常 数实部平均值分别提高到 19.07 和 15.91 ; 介电常 数虚部分别提高到 10.87 和 4.76; 介电损耗平均值
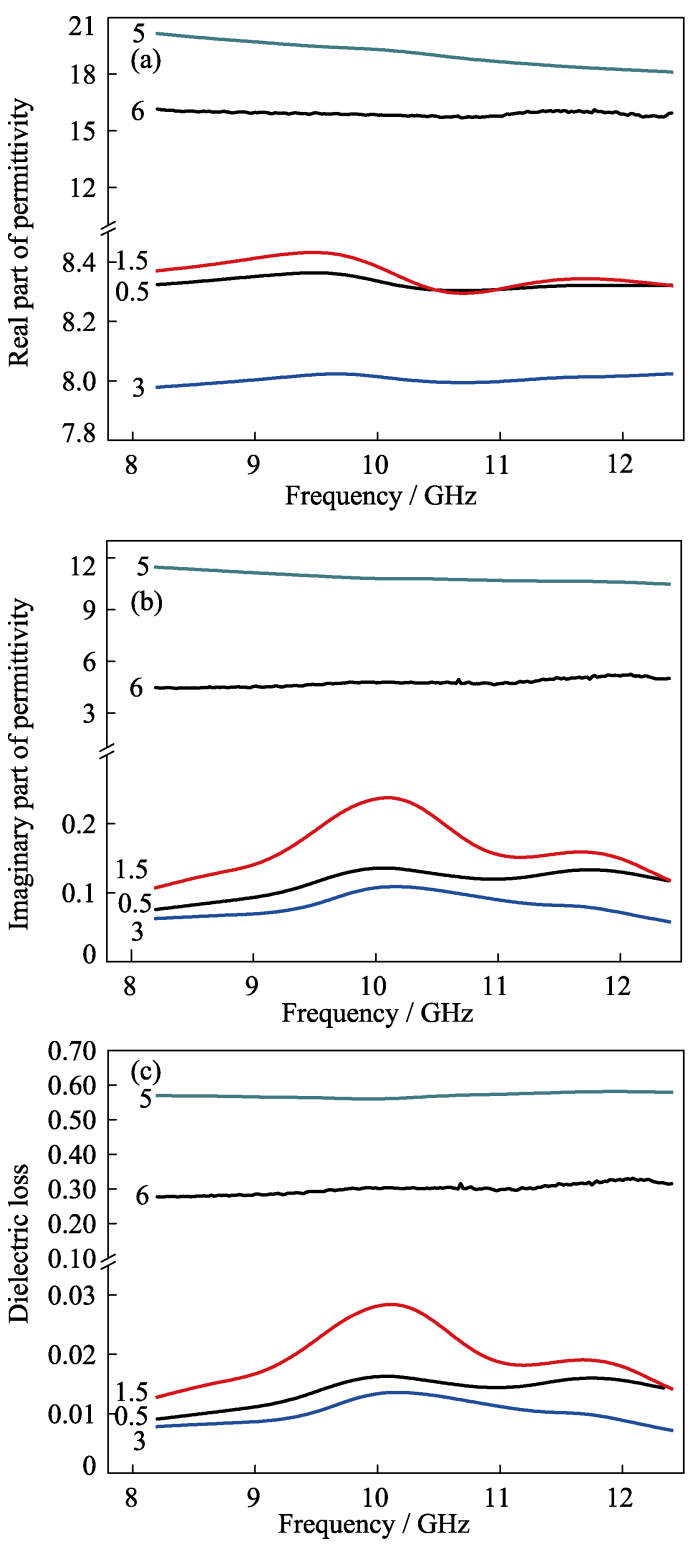

图 $71800^{\circ} \mathrm{C}$ 烧结的不同酚醛树脂含量复相陶瓷的 $\mathrm{X}$ 波段介 电性能

Fig. 7 Dielectric properties at X-band of composites with different phenolic resin contents sintered at $1800^{\circ} \mathrm{C}$

分别提高到 0.57 和 0.30 , 这表明材料具有良好的微 波衰减能力。

当酚醛树脂含量在 $3 \mathrm{wt} \%$ 以下时，此时材料中 的第二相处于 $\mathrm{Y}_{3} \mathrm{Al}_{5} \mathrm{O}_{12}$ 相向 $\mathrm{Y}_{2} \mathrm{O}_{3}$ 相转变的过程中, 残余碳含量不足，材料的性能与单一 AlN 陶瓷性能 相近，对电磁波的衰减能力很低。当酚醛树脂含量 提高后，残余碳过量，将会以碳膜的形式存在于材 料的气孔和氮化铝晶粒的三岔晶界处。碳膜的形成 能够在材料中引入大量的界面，在电磁场的作用下， 自由电荷由于在氮化铝晶粒和碳膜中的传导速率不 同，会在界面处发生积聚，从而形成强烈的弛豫极 化效应。并且碳膜的存在有助于材料中载流子的传 输, 增强材料的电导损耗能力, 最终使得材料的介 
电性能和介电损耗明显提升。

\section{3 结论}

1) 酚醛树脂形成的残余碳能够通过碳热还原 反应消除 AlN 颗粒表面的氧化层, 有效促进 AlN 陶 瓷的致密化进程, 降低烧结温度。当酚醛树脂含量 在 $3 \mathrm{wt} \%$ 以下时, 材料的致密度随着酚醛树脂含量 的增加显著提高, 当酚醛树脂含量提高到 $5 \mathrm{wt} \%$ 及 以上时, 此时残余碳含量较多, 材料的致密度开始 下降。

2) 材料中的第二相随着酚醛树脂添加量的增 加逐渐由 $\mathrm{Y}_{3} \mathrm{Al}_{5} \mathrm{O}_{12}$ 相向 $\mathrm{AlYO}_{3} 、 \mathrm{Al}_{2} \mathrm{Y}_{4} \mathrm{O}_{9}$ 和 $\mathrm{Y}_{2} \mathrm{O}_{3}$ 相转变。当酚醛树脂含量为 $6 \mathrm{wt} \%$ 时, 残余碳过量, 将以碳膜的形式存在于材料的气孔之中和氮化铝晶 粒的晶界处。

3) 当酚醛树脂含量为 $3 w t \%$ 以下时, 酚醛树脂 的添加能够有效提升材料的导热性能, 此时残余碳 将会消耗 AlN 颗粒表面的氧化层, 从而降低晶格氧 含量; 当其含量升高到 $5 \mathrm{wt} \%$ 时, 材料由于致密度下 降, 热导率也明显下降。而当其含量继续增加时, 由 于碳膜在气孔处的桥梁作用，材料的热导率没有明 显的恶化现象。

4) 酚醛树脂的添加能够明显提高材料的介电 性能，在 X 波段的介电损耗可高达 0.57 , 表明材料 具有良好的微波衰减能力, 此时材料的热导率为 $132.71 \mathrm{~W} /(\mathrm{m} \cdot \mathrm{K})$, 有望作为微波衰减材料应用于大 功率微波电真空器件中。

\section{参考文献:}

[1] BILJANA MIKIJELJ, DAVID K ABE, RON HUTCHEON. AINbased lossy ceramics for high average power microwave devices: performance-property correlation. Journal of the European Ceramic Society, 2003, 23(14): 2705-2709.

[2] SHI MING, LU YAN-PING. Development of AlN-based microwaveabsorbing materials. Bulletin of The Chinese Ceramic Society, 2005, 24(6): 76-79.
[3] SLACK GA, TANZILLI RA, POHL RO, et al. The intrinsic thermal conductivity of AlN. Journal of Physics and Chemistry of Solids, 1987, 48(7): 641-647.

[4] ZHANG YAN, MA HUI-NA, YANG ZHI-MING, et al. Research on dielectric properties of AlN-Mo composite ceramics. Materials Review, 2008, 22(12): 129-131.

[5] CHEN GUI-QIAO, LI XIAO-YUN, QIU TAI. Study on performance of AlN-W composites with microwave attenuation characteristics. China Ceramic Industry, 2006, 13(4): 22-25.

[6] YANG ZHEN-TAO, LU YAN-PING. Study on the preparation and properties of AlN-W microwave attenuation materials by pressureless sintering. Bulletin of The Chinese Ceramic Society, 2013, 32(9): 1741-1750.

[7] BU WEN-BO, QIU TAI, XU JIE. Preparation and microwave attenuation performance of AlN-SiC composites. Journal of the Chinese Ceramic Society, 2003, 31(9): 828-831.

[8] SERBENYUK T B, ALEKSANDROVA L I, ZAIKA M I, et al. Structure, mechanical and functional properties of aluminum nitride-silicon carbide ceramic material. Journal of Superhard Materials, 2008, 30(6): 384-391.

[9] BU WEN-BO, QIU TAI, XU JIE, et al. Study on microwave attenuation characteristics of AlN-C composites. Electronic Components \& Materials, 2003, 22(2): 1-3.

[10] WU HUA-ZHONG, XU QING-YAN, LI XIAO-YUN. Effect of attenuation agents on thermal conductivity of aluminum nitride attenuation ceramics. Ordnance Material Science and Engineering, 2009, 32(4): 35-37.

[11] LI XIU-QING. Present status of AIN ceramics package. Semiconductor Information, 1999, 36(2): 13-20.

[12] KIMBERLY A TRICK, TONY E SALIBA. Mechanisms of the pyrolysis of phenolic resin in a carbon/phenolic composite. Carbon, 1995, 33(11): 1509-1515.

[13] NAKAMATSU T, POMAR F, ISHIZAKI K. The effect of carbon coating of AlN powder on sintering behavior and thermal conductivity. Journal of Materials Science, 1999, 34(7): 1553-1556.

[14] MIODOWNIK MARK, HOLM ELIZABETH A, HASSOLD GREGORY N. Highly parallel computer simulations of particles pinning: Zener vindicated. Scripta Materialia, 2000, 42(12): 11731177.

[15] SLACK G A. Nonmetallic crystals with high thermal conductivity. Journal of Physics and Chemistry of Solids, 1973, 34(2): 321-335.

[16] LIU XIAO-FEI, ZHANG LI-TONG, YIN XIAO-WEI, et al. The microstructure of SiCN ceramics and their excellent electromagnetic wave absorbing properties. Ceramics International, 2015, 41(9): 11372-11378. 